\title{
ENTROPY STABILITY AND THE NO-SLIP WALL BOUNDARY CONDITION*
}

\author{
MAGNUS SVÄRD ${ }^{\dagger}$, MARK H. CARPENTER ${ }^{\ddagger}$, AND MATTEO PARSANI ${ }^{\S}$
}

\begin{abstract}
We present an entropy stable numerical scheme subject to no-slip wall boundary conditions. To enforce entropy stability only the no-penetration boundary condition and a temperature condition are needed at a wall, and this leads to an $L^{2}$ bound on the conservative variables. In this article, we take the next step and design a finite difference scheme that also bounds the velocity gradients. This necessitates the use of the full no-slip conditions.
\end{abstract}

Key words. Navier-Stokes, finite difference, nonlinear stability, wall boundary conditions

AMS subject classifications. 65M06, 65M12, 35L65, 76N15

DOI. $10.1137 / 16 \mathrm{M} 1097225$

1. Introduction. In recent years, there has been increasing interest in entropy stable numerical schemes for the compressible Euler and Navier-Stokes equations. Entropy stability leads to an $L^{2}$ estimate of the conservative variables, which enhances the robustness of the method for strongly nonlinear flows. There is at present a wide variety of schemes that enforce entropy stability; see, e.g., [Tad03, Svä12, FC13, PCN15, $\left.\mathrm{CFN}^{+} 16\right]$. Entropy stability was first introduced as a constraint for internal stencils, but lately these schemes have been developed to include boundary conditions. (See [SM12, SO14, PCN15].)

This article is a contribution towards the nonlinear treatment of no-slip wall boundary conditions for the compressible Navier-Stokes equations. At walls, it is well known from linear well-posedness theory that four boundary conditions are needed in three space dimensions. (See [KL89, SN08].) These are no-slip, i.e., all three velocity components are zero, and one temperature condition. The latter can be a constant temperature at the wall or the adiabatic condition, i.e., the normal gradient of the temperature is zero. ${ }^{1}$

Entropy stability formally resembles the well-known energy stability analysis used for linear (and linearized) schemes and requires a summation-by-parts (SBP) property, [SN08]. At a viscous wall, the entropy analysis on a bounded domain requires a temperature condition and the no-penetration condition. In three dimensions, it means that only two out of the four boundary conditions that the linear stability analysis stipulates are required. (See [PCN15].) The reason for this is that entropy stability does not imply well-posedness. Another necessary bound for a nonlinear

\footnotetext{
* Received by the editors October 4, 2016; accepted for publication (in revised form) October 11, 2017; published electronically January 18, 2018.

http://www.siam.org/journals/sinum/56-1/M109722.html

Funding: This work was partially supported by King Abdullah University of Science and Technology (KAUST) in Thuwal, Saudi Arabia.

${ }^{\dagger}$ Department of Mathematics, University of Bergen, 5020 Bergen, Norway (Magnus.Svard@ uib.no).

${ }^{\ddagger}$ Computational AeroScience Branch, NASA Langley Research Center (LaRC), Hampton, VA 23681 (mark.h.carpenter@nasa.gov).

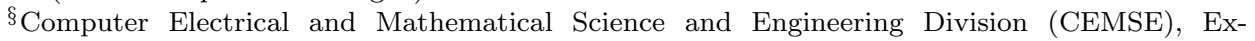
treme Computing Research Center (ECRC), King Abdullah University of Science and Technology (KAUST), Thuwal 23955-6900, Saudi Arabia (matteo.parsani@kaust.edu.sa).

${ }^{1}$ We will also use the term no-penetration condition, which means that only the normal component of the velocity is set to zero.
} 
well-posedness theory is a bound on velocity gradients. ${ }^{2}$ (See, e.g., [FV10].) Such a bound requires the use of the full no-slip condition; i.e., the tangential components of the velocity, in addition to the normal component, must be zero.

In this article, we propose a finite difference scheme for the compressible NavierStokes equations with boundary schemes that enforce the no-slip and adiabatic temperature conditions. We build on the work in [PCN15], and in addition to proving a bound on the entropy, we also demonstrate that the velocity gradients are bounded.

Naturally, adding more constraints will reduce the number of possible schemes. It turns out that the difference approximations must satisfy a discrete Leibniz rule. Our results should be readily generalizable to other finite difference schemes that have this property.

The general technique used to derive the velocity gradient bound should also be applicable to other types of schemes, such as entropy stable discontinuous Galerkin and spectral collocation schemes $\left[\mathrm{CFN}^{+} 16, \mathrm{PCFN} 16\right]$. These schemes do not automatically bound the velocity gradients and hence do not formally need the full no-slip conditions to be entropy stable. However, the nonlinear nature of the analysis presented herein makes broad generalizations impossible. The analysis has to be worked out for each individual scheme in a similar way.

2. The continuous framework. The compressible Navier-Stokes equations are

$$
\mathbf{u}_{t}+\mathbf{f}_{x}+\mathbf{g}_{y}+\mathbf{h}_{z}=\mathbb{F}_{x}+\mathbb{G}_{y}+\mathbb{H}_{z} \quad \text { on } \quad \Omega=(0,1)^{3}, \quad 0<t<\mathcal{T},
$$

where

$$
\begin{aligned}
& \mathbf{u}=\left(\rho, \rho v_{1}, \rho v_{2}, \rho v_{3}, E\right)^{T}, \\
& \mathbf{f}=\left(\rho v_{1}, \rho v_{1}^{2}+p, \rho v_{1} v_{2}, \rho v_{1} v_{3}, v_{1}(E+p)\right)^{T}, \\
& \mathbf{g}=\left(\rho v_{2}, \rho v_{2} v_{1}, \rho v_{2}^{2}+p, \rho v_{2} v_{3}, v_{2}(E+p)\right)^{T}, \\
& \mathbf{h}=\left(\rho v_{3}, \rho v_{3} v_{1}, \rho v_{3} v_{2}, \rho v_{3}^{2}+p, v_{3}(E+p)\right) .{ }^{T}
\end{aligned}
$$

The conservative variables are density $\rho$; momentum $\rho v_{1}, \rho v_{2}, \rho v_{3}$; and the total energy $E=\frac{1}{2} \rho|\mathbf{v}|^{2}+\rho e . p$ is the pressure, $T$ the temperature, and $\mathbf{v}$ the velocity vector. Furthermore, $e=c_{v} T$ is the internal energy and $\rho e=\frac{p}{\gamma-1} . R$ is the gas constant and $\gamma=c_{p} / c_{v}$.

The viscous fluxes are given as

$$
\begin{aligned}
& \mathbb{F}=\left(0, \tau_{x x}, \tau_{x y}, \tau_{x z}, v_{1} \tau_{x x}+v_{2} \tau_{x y}+v_{3} \tau_{x z}+\kappa T_{x}\right), \\
& \mathbb{G}=\left(0, \tau_{y x}, \tau_{y y}, \tau_{y z}, v_{1} \tau_{y x}+v_{2} \tau_{y y}+v_{3} \tau_{y z}+\kappa T_{y}\right), \\
& \mathbb{H}=\left(0, \tau_{z x}, \tau_{z y}, \tau_{z z}, v_{1} \tau_{z x}+v_{2} \tau_{z y}+v_{3} \tau_{z z}+\kappa T_{z}\right),
\end{aligned}
$$

where $\tau_{i j}=-\frac{2}{3} \mu v_{k, k} \delta_{i j}+\mu\left(v_{i, j}+v_{j, i}\right)$. The dynamic viscosity is $\mu(T) \geq \mu_{0}>0$, and the heat conductivity is $\kappa(T) \geq \kappa_{0}>0$.

The equations (1) are augmented with the (wall) boundary conditions,

$$
\begin{aligned}
v_{k} & =0, \quad k=1,2,3 \quad \text { no-slip } \\
\frac{1}{T} \frac{\partial T}{\partial n} & =0, \quad \text { adiabatic condition }
\end{aligned}
$$

on $\partial \Omega$. $n$ denotes the normal direction. Furthermore, we will refer to the normal component of the no-slip condition as the no-penetration condition.

\footnotetext{
${ }^{2}$ As is well known, no complete well-posedness theory is available for the Navier-Stokes equations. Nevertheless, some bounds are clearly indispensable.
} 
Remark. The adiabatic condition (3) is usually given as $\partial_{n} T=0$. Here, we state this form since it is how it appears in the entropy estimate, as we will see below.

Associated with (1) is the entropy $U(\mathbf{u})=-\rho S$ (a convex function), where the specific entropy is $S=\log \left(p / \rho^{\gamma}\right)$, and the entropy fluxes are $F(\mathbf{u})=-\rho v_{1} S, G(\mathbf{u})=$ $-\rho v_{2} S, H(\mathbf{u})=-\rho v_{3} S$ that satisfy

$$
U^{\prime} \mathbf{f}^{\prime}=F^{\prime}, \quad U^{\prime} \mathbf{g}^{\prime}=G^{\prime}, \quad U^{\prime} \mathbf{h}^{\prime}=H^{\prime} .
$$

Furthermore, the so-called entropy variables are

$$
U_{\mathbf{u}}=\mathbf{q}^{T}=\frac{1}{c_{v}}\left(c_{v}(\gamma-S)-\frac{u^{2}+v^{2}+w^{2}}{T}, \frac{u}{T}, \frac{v}{T}, \frac{w}{T}, \frac{-1}{T}\right) .
$$

The idea of entropy stability is to discretely mimic an entropy inequality that is valid for the continuum equations. To obtain the continuum entropy inequality, we premultiply (1) by $\mathbf{q}^{T}$, integrate in space, and assume that $\rho \geq 0$. Using (4), we arrive at

$$
\int_{\Omega}\left(U_{t}+F_{x}+G_{y}+H_{z}\right) d \mathbf{x}=\int_{\Omega} \mathbf{q}^{T}\left(\mathbb{F}_{x}+\mathbb{G}_{y}+\mathbb{H}_{z}\right) d \mathbf{x}
$$

To reduce notation we consider only the boundary terms at $x=0$ and keep in mind that the others can be treated similarly. Hence, after partial integration, we have

$$
\int_{\Omega} U_{t} d \mathbf{x}-\int_{x=0, \partial \Omega} F(\mathbf{u}) d y d z=\int_{x=0, \partial \Omega} \mathbf{q}^{T} \mathbb{F} d y d z-\int_{\Omega}\left(\mathbf{q}_{x}^{T} \mathbb{F}+\mathbf{q}_{y}^{T} \mathbb{G}+\mathbf{q}_{z}^{T} \mathbb{H}\right) d \mathbf{x} .
$$

Using the no-penetration boundary condition $v_{1}=0$ (normal velocity at $x=0$ ), the $F(\mathbf{u}(x=0))$ term vanishes. Using (3), we obtain

$$
\int_{x=0, \partial \Omega} \mathbf{q}^{T} \mathbb{F} d y d z=-\kappa \frac{T_{x}}{T}=0
$$

where we have used (3) noting that the normal direction and the $x$-axis are aligned at $x=0$. We arrive at the estimate

$$
\int_{\Omega} U_{t} d \mathbf{x}+\int_{\Omega}\left(\mathbf{q}_{x}^{T} \mathbb{F}+\mathbf{q}_{y}^{T} \mathbb{G}+\mathbf{q}_{z}^{T} \mathbb{H}\right) d \mathbf{x}=0
$$

The second integral in (8) is positive and expresses diffusion of entropy due to the viscosity and heat diffusion.

Equation (8) is the entropy estimate, and we emphasize that the full no-slip boundary conditions were not necessary in its derivation. (We never used $v_{2}=v_{3}=0$ at $x=0$.) This was observed in [PCN15], and since it is well known (from linear theory) that the compressible Navier-Stokes equations require the full no-slip condition, the authors incorporated the no-slip condition in an entropy consistent manner in their scheme.

We demonstrate the procedure to obtain a velocity gradient bound in the twodimensional continuous case (to reduce notation). The same technique will be applied to our discrete scheme below. 
From (8), we have a bound on the following quantity (in two dimensions):

$$
\begin{array}{r}
C=-\int_{\Omega} U(\mathbf{x}, t) d \mathbf{x}+\int_{\Omega} U(\mathbf{x}, 0) d \mathbf{x}=\int_{0}^{\mathcal{T}} \int_{\Omega}\left(\mathbf{q}_{x}^{T} \mathbb{F}+\mathbf{q}_{y}^{T} \mathbb{G}\right) d \mathbf{x} d t \\
=\int_{0}^{\mathcal{T}} \int_{\Omega}\left(\frac{v_{1}}{T}\right)_{x} \tau_{x x}+\left(\frac{v_{2}}{T}\right)_{x} \tau_{x y}-\left(\frac{1}{T}\right)_{x}\left(v_{1} \tau_{x x}+v_{2} \tau_{x y}\right) \\
+\left(\frac{v_{1}}{T}\right)_{y} \tau_{x y}+\left(\frac{v_{2}}{T}\right)_{y} \tau_{y y}-\left(\frac{1}{T}\right)_{y}\left(v_{1} \tau_{x y}+v_{2} \tau_{y y}\right) d \mathbf{x} d t \\
+\int_{0}^{\mathcal{T}} \int_{\Omega} \kappa \frac{T_{x}^{2}+T_{y}^{2}}{T^{2}} d \mathbf{x} d t .
\end{array}
$$

Both integrals in the last expression are positive and are therefore bounded independently by $C$. Using the Leibniz rule on the first integral results in

$$
C>\int_{0}^{\mathcal{T}} \int_{\Omega} \frac{1}{T}\left(\left(v_{1}\right)_{x} \tau_{x x}+\left(v_{2}\right)_{x} \tau_{x y}+\left(v_{1}\right)_{y} \tau_{x y}+\left(v_{2}\right)_{y} \tau_{y y}\right) d \mathbf{x} d t
$$

By assuming that temperature is bounded from above, i.e., there is a $T_{\max } \geq T>0$ for all $\mathbf{x} \in \Omega$, we have

$$
C>\int_{0}^{\mathcal{T}} \frac{1}{T_{\max }} \int_{\Omega}\left(\left(v_{1}\right)_{x} \tau_{x x}+\left(v_{2}\right)_{x} \tau_{x y}+\left(v_{1}\right)_{y} \tau_{x y}+\left(v_{2}\right)_{y} \tau_{y y}\right) d \mathbf{x} d t
$$

If we can rephrase the integrand as a convex combination of all velocity gradients, we have obtained the desired bound. To this end, we insert the definitions of $\tau_{i j}$ (and change the notation $v_{1}=u, v_{2}=v$ to reduce the number of indices):

$$
\begin{array}{r}
C T_{\max }>\int_{0}^{T} \int_{\Omega}\left(u_{x} \tau_{x x}+v_{x} \tau_{x y}+u_{y} \tau_{x y}+v_{y} \tau_{y y}\right) d \mathbf{x} d t \\
=\int_{0}^{T} \int_{\Omega}\left(u_{x}\left(\frac{4}{3} u_{x}-\frac{2}{3} v_{y}\right)+\left(v_{x}+u_{y}\right)\left(v_{x}+u_{y}\right)+v_{y}\left(\frac{4}{3} v_{y}-\frac{2}{3} u_{x}\right)\right) d \mathbf{x} d t \\
=\int_{0}^{T} \int_{\Omega}\left(u_{x}\left(\frac{4}{3} u_{x}-\frac{2}{3} v_{y}\right)+v_{x}^{2}+u_{y}^{2}+2 v_{x} u_{y}+v_{y}\left(\frac{4}{3} v_{y}-\frac{2}{3} u_{x}\right)\right) d \mathbf{x} d t
\end{array}
$$

Next, we take a closer look at one of the terms in the last expression:

$$
\begin{array}{r}
\int_{\Omega} v_{x} u_{y} d \mathbf{x}=\left.\int_{\partial \Omega} u_{y} v\right|_{x=0} ^{1} d y d z-\int_{\Omega} u_{x y} v d \mathbf{x} \\
=\left.\int_{\partial \Omega} u_{y} v\right|_{x=0} ^{1} d y d z-\left.\int_{\partial \Omega} u_{x} v\right|_{y=0} ^{1} d x d z+\int_{\Omega} u_{x} v_{y} d \mathbf{x} .
\end{array}
$$

To cancel the $x$-boundary terms, we need $v=0$, i.e., the tangential component of the no-slip boundary condition that was not used in the entropy estimate. Finally,

$$
\begin{array}{r}
C T_{\max }>\int_{0}^{T} \int_{\Omega}\left(u_{x} \tau_{x x}+v_{x} \tau_{x y}+u_{y} \tau_{x y}+v_{y} \tau_{y y}\right) d \mathbf{x} d t \\
=\int_{0}^{T} \int_{\Omega}\left(u_{x}\left(\frac{4}{3} u_{x}-\frac{2}{3} v_{y}\right)+v_{x}^{2}+u_{y}^{2}+2 v_{y} u_{x}+v_{y}\left(\frac{4}{3} v_{y}-\frac{2}{3} u_{x}\right)\right) d \mathbf{x} d t \\
=\int_{0}^{T} \int_{\Omega}\left(\frac{4}{3} u_{x}^{2}+v_{x}^{2}+u_{y}^{2}+\frac{2}{3} v_{y} u_{x}+\frac{4}{3} v_{y}^{2}\right) d \mathbf{x} d t \\
=\int_{0}^{T} \int_{\Omega}\left(u_{x}^{2}+v_{x}^{2}+u_{y}^{2}+\frac{1}{3}\left(v_{y}+u_{x}\right)^{2}+v_{y}^{2}\right) d \mathbf{x} d t .
\end{array}
$$

Copyright $\odot$ by SIAM. Unauthorized reproduction of this article is prohibited. 
If $C$ is a bounded constant (this will be proved below), the last expression constitutes a bound on the velocity gradients. To obtain this bound, the no-slip boundary condition was needed.

Furthermore, we made two assumptions that we will use throughout the paper:

1. The density and temperature remain bounded away from 0 , i.e., they have strictly positive minima. (This is necessary for the entropy estimate.)

2. The temperature remains bounded at all points in time and space, i.e., it has a finite maximum. (This is necessary for the $\nabla \cdot \mathbf{v}$ estimate.)

3. The semidiscrete entropy analysis. In this section, we introduce a finite difference scheme and carry out the discrete counterpart of the above derivation. This will lead to a proof of our main result, Theorem 8.

3.1. Definition of the numerical scheme. We discretize the domain $\Omega$ with $N+1$ points in the $x, y, z$-directions. Then we introduce $h=1 / N$ and $x_{i}=i h$, $y_{j}=j h$, and $z_{k}=k h, i, j, k=0, \ldots, N$. The discrete spatial domain is denoted by $\Omega_{N}$. The semidiscrete (continuous in time) conservative variables are $\mathbf{u}_{i j k}=$ $\left(\rho_{i j k}, m_{i j k}^{1}, m_{i j k}^{2}, m_{i j k}^{3}, E_{i j k}\right)^{T}$, where the components are the numerical variables corresponding to density, momentum in the $x, y, z$-directions, and total energy. We use $u_{i j k}^{k}$ to denote the $k$ th component of $\mathbf{u}_{i j k}$, e.g., $u_{i j k}^{1}=\rho_{i j k}$. All variables satisfy the same algebraic relations as their continuous counterparts. For example, $E_{i j k}=\frac{p_{i j k}}{\gamma-1}+\frac{1}{2 \rho_{i j k}}\left(\left(m_{i j k}^{1}\right)^{2}+\left(m_{i j k}^{2}\right)^{2}+\left(m_{i j k}^{3}\right)^{2}\right)$. To simplify notation, we will use $u_{i j k}, v_{i j k}$, and $w_{i j k}$ to denote the velocity components.

The difference operators we will use are given by

$$
\begin{aligned}
D^{x} a_{i j k} & =\frac{a_{i+1 j k}-a_{i-1 j k}}{2 h}, \\
D^{x} a_{0 j k} & =\frac{a_{1 j k}-a_{0 j k}}{h}\left(=D_{+h}^{x} a_{0 j k}\right), \quad 0<i<N, \\
D^{x} a_{N j k} & =\frac{a_{N j k}-a_{(N-1) j k}}{h}\left(=D_{-h}^{x} a_{N j k}\right),
\end{aligned}
$$

where $a$ can be a scalar or a vector. In the latter case, the operation is carried out componentwise. To be able to write the scheme compactly, we do not change the notation for the boundary operator. (However, the notation in the parentheses above is sometimes used to highlight that it is the skew boundary operator.)

Furthermore,

$$
\begin{aligned}
D_{-}^{x} a_{i+1 / 2 j k} & =\frac{a_{i+1 / 2 j k}-a_{i-1 / 2 j k}}{h}, \\
D_{-}^{x} a_{1 / 2 j k} & =\frac{a_{1 / 2 j k}-a_{0 j k}}{h / 2}, \\
D_{-}^{x} a_{N+1 / 2 j k} & =\frac{a_{N j k}-a_{N-1 / 2 j k}}{h / 2} .
\end{aligned}
$$

The operators in the $y$ - and $z$-directions are defined analogously.

Central in the analysis are also the following Leibniz (or product) rules:

$$
\begin{aligned}
\frac{a_{i+1} b_{i+1}-a_{i-1} b_{i-1}}{h} & =\frac{a_{i+1}+a_{i-1}}{2} \frac{b_{i+1}-b_{i-1}}{h}+\frac{b_{i+1}+b_{i-1}}{2} \frac{a_{i+1}-a_{i-1}}{h}, \\
\frac{a_{i+1} b_{i+1}-a_{i} b_{i}}{h} & =a_{i+1} \frac{b_{i+1}-b_{i}}{h}+b_{i} \frac{a_{i+1}-a_{i}}{h} .
\end{aligned}
$$

Copyright (c) by SIAM. Unauthorized reproduction of this article is prohibited. 
In the interior of the domain and along (but not perpendicular to) boundaries, we define

$$
\begin{aligned}
\mathbf{f}_{i+1 / 2 j k} & =\mathbf{f}^{\mathbf{e c}}{ }_{i+1 / 2 j k}\left(\mathbf{u}_{i+1 j k}, \mathbf{u}_{i j k}\right), \\
\mathbf{g}_{i j+1 / 2 k} & =\mathbf{f}^{\mathbf{e c}}{ }_{i j+1 / 2 k}\left(\mathbf{u}_{i j+1 k}, \mathbf{u}_{i j k}\right), \\
\mathbf{h}_{i j k+1 / 2} & =\mathbf{f}^{\mathbf{e c}}{ }_{i j k+1 / 2}\left(\mathbf{u}_{i j k+1}, \mathbf{u}_{i j k}\right),
\end{aligned}
$$

where $\mathbf{f}^{\mathbf{e c}}\left(\mathbf{u}_{1}, \mathbf{u}_{2}\right)$ is an entropy conservative flux between two states. For instance, $\mathbf{f}^{\mathbf{e c}}$ can be the flux defined in [IR09]. (This flux can be replaced by any entropy stable flux; see [Tad03]. The entropy conservative choice renders the convective terms to be second-order accurate while a first-order diffusion (e.g., Lax-Friedrichs or Roe) would be entropy stable but only first-order accurate.)

The fluxes on the boundary are computed using boundary data. At the boundary $x=0$, we define

$$
\mathbf{f}_{0 j k}=\mathbf{f}^{\mathbf{e c}}\left(\mathbf{u}_{0 j k}, \mathfrak{g}_{0 j k}\right) .
$$

At the boundary $\mathbf{f}^{\mathbf{e c}}$ has to be an entropy conservative flux. The data vector $\mathfrak{g}$ is constructed from $\mathbf{u}$ by changing the sign of $m^{1}$, i.e.,

$$
\mathfrak{g}_{0 j k}=\left(\rho_{0 j k},-m_{0 j k}^{1}, m_{0 j k}^{2}, m_{0 j k}^{3}, E_{0 j k}\right)^{T} .
$$

We emphasize that (16) and (17) enforce the no-penetration condition at $x=0$. (See [PCN15].)

Remark. In [PCN15] this boundary condition was stated as an equivalent forcing term, a so-called simultaneous approximation term (SAT), on the boundary. The definition, ((16) and (17)), is exactly equivalent to [PCN15]. (See also [SO14] for a similar way to enforce this boundary condition.)

Next, we turn to the definition of the diffusive fluxes. We will use the following shorthand notation:

$$
\begin{array}{ll}
D^{x} u_{i j k}=\left(\mathfrak{u}_{x}\right)_{i j k}, & 0 \leq i, j, k \leq N, \\
D^{y} u_{i j k}=\left(\mathfrak{u}_{y}\right)_{i j k}, & 0 \leq i, j, k \leq N, \\
D^{z} u_{i j k}=\left(\mathfrak{u}_{z}\right)_{i j k}, & 0 \leq i, j, k \leq N .
\end{array}
$$

(Recall that perpendicular to the boundaries, the differences are skew; i.e., $D^{x} u_{0 j k}=$ $D_{+h}^{x} u_{0 j k}=\left(\mathfrak{u}_{x}\right)_{0 j k}$.) We use the same convention for the temperature derivatives. For example,

$$
D^{x} T_{i j k}=\left(\mathfrak{T}_{x}\right)_{i j k}, \quad 0 \leq i, j, k \leq N
$$

Define

$$
\begin{aligned}
\left(\tau_{x x}\right)_{i j k} & =\mu\left(\frac{4}{3}\left(\mathfrak{u}_{x}\right)_{i j k}-\frac{2}{3}\left(\left(\mathfrak{v}_{y}\right)_{i j k}+\left(\mathfrak{w}_{z}\right)_{i j k}\right)\right), \\
\left(\tau_{x y}\right)_{i j k} & =\left(\tau_{y x}\right)_{i j k}=\mu\left(\left(\mathfrak{u}_{y}\right)_{i j k}+\left(\mathfrak{v}_{x}\right)_{i j k}\right), \\
\left(\tau_{x z}\right)_{i j k} & =\left(\tau_{z x}\right)_{i j k}=\mu\left(\left(\mathfrak{u}_{z}\right)_{i j k}+\left(\mathfrak{w}_{x}\right)_{i j k}\right), \\
\left(\tau_{y y}\right)_{i j k} & =\mu\left(\frac{4}{3}\left(\mathfrak{v}_{y}\right)_{i j k}-\frac{2}{3}\left(\left(\mathfrak{u}_{x}\right)_{i j k}+\left(\mathfrak{w}_{z}\right)_{i j k}\right)\right), \\
\left(\tau_{y z}\right)_{i j k} & =\left(\tau_{z y}\right)_{i j k}=\mu\left(\left(\mathfrak{v}_{z}\right)_{i j k}+\left(\mathfrak{w}_{y}\right)_{i j k}\right), \\
\left(\tau_{z z}\right)_{i j k} & =\mu\left(\frac{4}{3}\left(\mathfrak{w}_{z}\right)_{i j k}-\frac{2}{3}\left(\left(\mathfrak{u}_{x}\right)_{i j k}+\left(\mathfrak{v}_{y}\right)_{i j k}\right)\right) .
\end{aligned}
$$

Copyright (c) by SIAM. Unauthorized reproduction of this article is prohibited. 
Furthermore, we will need arithmetic averages and we use the following notation:

$$
\stackrel{x}{a_{i j k}}=\left(a_{i+1 j k}+a_{i-1 j k}\right) / 2
$$

where $x, y, z$ signifies the index that is averaged. We will apply (18) to $1 / T$ and the velocities. At the boundary, $I=0$, we use the same notation but do not take the average:

$$
\begin{aligned}
\stackrel{x}{u_{0 j k}} & =u_{0 j k}, \\
& { }^{x} \\
T^{-1}{ }_{0 j k} & =\left(T^{-1}\right)_{1 j k} .
\end{aligned}
$$

The viscous fluxes are split into two parts, a viscous and a heat diffusive flux. In the $x$-direction, we write

$$
\mathbf{F}_{i j k}=\mathbf{F}_{i j k}^{\mu}+\mathbf{F}_{i j k}^{\kappa} .
$$

The viscous fluxes (excluding the heat flux) are discretized as

$$
\begin{gathered}
\mathbf{F}_{i, j k}^{\mu}=1_{x}\left(0, \tau_{x x}, \tau_{x y}, \tau_{x z}, \stackrel{x}{u}_{i j k} \tau_{x x}+\stackrel{x}{v}_{i j k} \tau_{x y}+\stackrel{x}{w}_{i j k} \tau_{x z}\right), \\
\mathbf{G}_{i j k}^{\mu}=1_{y}\left(0, \tau_{x y}, \tau_{y y}, \tau_{y z}, \stackrel{y}{u}_{i j k} \tau_{x y}+\stackrel{y}{v}_{i j k} \tau_{y y}+\stackrel{y}{w}_{i j k} \tau_{y z}\right), \\
\mathbf{H}_{i, j, k}^{\mu}=1_{z}\left(0, \tau_{z x}, \tau_{z y}, \tau_{z z}, \stackrel{z}{u_{i j k}} \tau_{z x}+\stackrel{z}{v}_{i j k} \tau_{z y}+\stackrel{z}{w}_{i j k} \tau_{z z}\right),
\end{gathered}
$$

where the $\tau$ 's are all evaluated at $i j k$. Furthermore,

$$
\stackrel{x}{\stackrel{x}{1}} 1_{x}=T_{i j k}^{-1} / T^{-1 j k}, \quad 1_{y}=T_{i j k}^{-1} /\left(T^{-1}\right)_{i j k}, \quad 1_{z}=T_{i j k}^{-1} /\left(T^{-1}\right)_{i j k}
$$

are accurate approximations of 1 .

The heat fluxes are defined as

$$
\begin{aligned}
\mathbf{F}_{i j k}^{\kappa} & =\left(0,0,0, \kappa\left(\mathfrak{T}_{x}\right)_{i j k}\right)^{T}, \\
\mathbf{G}_{i j k}^{\kappa} & =\left(0,0,0, \kappa\left(\mathfrak{T}_{y}\right)_{i j k}\right)^{T}, \\
\mathbf{H}_{i j k}^{\kappa} & =\left(0,0,0, \kappa\left(\mathfrak{T}_{z}\right)_{i j k}\right)^{T} .
\end{aligned}
$$

Finally, a second-order accurate scheme approximating (1) takes the form

$$
\begin{aligned}
\left(\mathbf{u}_{i j k}\right)_{t} & +D_{-}^{x} \mathbf{f}_{i+1 / 2 j k}+D_{-}^{y} \mathbf{g}_{i j+1 / 2 k}+D_{-}^{z} \mathbf{h}_{i j k+1 / 2} \\
& =D^{x} \mathbf{F}_{i j k}+D^{y} \mathbf{G}_{i j k}+D^{z} \mathbf{H}_{i j k}+S A T_{i j k}, \quad 0 \leq i, j, k \leq N .
\end{aligned}
$$

Here, $S A T_{i j k}$ enforces the boundary conditions associated with the diffusive fluxes. (Recall that the inviscid boundary condition is built into the numerical flux (16).) We split it as $S A T_{i j k}=S A T_{i j k}^{\kappa}+S A T_{i j k}^{\mu}$, where, for the $x=0$ boundary,

$$
\begin{aligned}
S A T_{0 j k}^{\mu} & =\sigma \mu\left(0, u_{0 j k}, v_{0 j k}, w_{0 j k}, 0\right)=\sigma \frac{\mu}{T_{0 j k}} S A T_{0 j k}^{n o-s l i p}, \\
S A T_{0 j k}^{n o-s l i p} & =T_{0 j k}(0, u-0, v-0, w-0,0)_{0 j k}^{T}, \\
\sigma & \leq-\frac{1}{3 p_{0} h}
\end{aligned}
$$

enforce the boundary conditions (2). (The "-0" signifies that $u, v, w$ are penalized against zero on the boundary.) Furthermore,

$$
S A T_{0 j k}^{\kappa}=-\left(\mathbf{F}_{0 j k}^{\kappa}-\mathbf{0}\right)
$$

enforces the condition (3). 
3.2. The semidiscrete entropy estimate. To carry out the the entropy analysis, we need a number of preliminary results. The first is an expression for $D^{x} \mathbf{q}_{i j k}$. Using the discrete Leibniz rule (15), we have

$$
D^{x} \mathbf{q}_{i j k}=\frac{1}{c_{v}}\left(\begin{array}{c}
c_{v} D^{x} q_{i j k}^{1} \\
D^{x} \frac{u_{i j k}}{T_{i j k}} \\
D^{x} \frac{v_{i j k}}{T_{i j k}} \\
D^{x} \frac{w_{i j k}}{T_{i j k}} \\
D^{x} \frac{-1}{T_{i j k}}
\end{array}\right)=\frac{1}{c_{v}}\left(\begin{array}{c}
c_{v} D_{x} q_{i j k}^{1} \\
T^{-1}{ }_{i j k} D^{x} u_{i j k}+\stackrel{x}{u}_{i j k} D^{x} \frac{1}{T_{i j k}} \\
x \\
T^{-1}{ }_{i j k} D^{x} v_{i j k}+\stackrel{x}{v}_{i j k} D^{x} \frac{1}{T_{i j k}} \\
x \\
T^{-1}{ }_{i j k} D^{x} w_{i j k}+\mathbf{w}_{i j k} D^{x} \frac{1}{T_{i j k}} \\
D^{x} \frac{-1}{T_{i j k}}
\end{array}\right) .
$$

Note in particular the specific rule at the boundary, e.g.,

$$
D_{+h}^{x} \frac{u_{0 j k}}{T_{0 j k}}=\stackrel{x}{-1} T_{0 j k} D_{+h}^{x} u_{i j k}+\stackrel{x}{u_{0 j k}} D_{+h}^{x} \frac{1}{T_{i j k}} .
$$

Remark. $D^{x} q^{1}$ will not affect the subsequent calculations, and we omit its precise form here.

To make the derivation of an entropy estimate from (21) more tractable, we summarize some of the work in a few lemmas. Lemma 1 states that the discrete counterparts of the heat diffusion terms in (8) are indeed positive.

LEMma 1. If $T_{i j k}$ are nonnegative for all $0 \leq i, j, k \leq N$, then

$$
\begin{aligned}
& \left(D^{x} \mathbf{q}_{i j k}\right)^{T} \mathbf{F}_{i j k}^{\kappa} \geq 0, \quad 1 \leq i \leq N-1, \quad 0 \leq j, k \leq N, \\
& \left(D^{y} \mathbf{q}_{i j k}\right)^{T} \mathbf{G}_{i j k}^{\kappa} \geq 0, \quad 1 \leq j \leq N-1, \quad 0 \leq i, k \leq N, \\
& \left(D^{z} \mathbf{q}_{i j k}\right)^{T} \mathbf{H}_{i j k}^{\kappa} \geq 0, \quad 1 \leq k \leq N-1, \quad 0 \leq i, j \leq N .
\end{aligned}
$$

Furthermore, at the boundaries, analogous statements hold:

$$
\begin{array}{ll}
\left(D_{+h}^{x} \mathbf{q}_{i j k}\right)^{T} \mathbf{F}_{0 j k}^{\kappa} \geq 0, & 0 \leq j, k \leq N, \\
\left(D_{+h}^{y} \mathbf{q}_{i j k}\right)^{T} \mathbf{G}_{0 j k}^{\kappa} \geq 0, & 0 \leq i, k \leq N, \\
\left(D_{+h}^{z} \mathbf{q}_{i j k}\right)^{T} \mathbf{H}_{0 j k}^{\kappa} \geq 0, & 0 \leq i, j \leq N .
\end{array}
$$

Proof. The heat fluxes are defined as

$$
\mathbf{F}_{i j k}^{\kappa}=\left(0,0,0, \kappa D^{x} T_{i j k}\right)^{T} .
$$

Suppressing the $j k$ indices, we have $D^{x} T_{i}=T_{i+1} T_{i-1} D^{x} \frac{-1}{T_{i}}$. Then

$$
c_{v}\left(D^{x} \mathbf{q}_{i j k}\right)^{T} \mathbf{F}_{i j k}^{\kappa}=\kappa T_{i+1 j k} T_{i-1 j k}\left(D^{x} \frac{-1}{T_{i j k}}\right)^{2} \geq 0 .
$$

The other statements are proved analogously. At the boundary, we use $D_{+h}^{x} T_{i}=$ $T_{i+1} T_{i} D_{+}^{x} \frac{-1}{T_{i}}$, and the results follow.

Next, we show that the discrete viscous terms corresponding to (8) are positive.

LEMma 2. If $T_{i j k}$ is nonnegative, then

$$
\left(D^{x} \mathbf{q}_{i j k}\right)^{T} \mathbf{F}_{i j k}^{\mu}+\left(D^{y} \mathbf{q}_{i j k}\right)^{T} \mathbf{G}_{i j k}^{\mu}+\left(D^{z} \mathbf{q}_{i j k}\right)^{T} \mathbf{H}_{i j k}^{\mu} \geq 0, \quad 0 \leq i, j, k \leq N .
$$

Copyright $@$ ㅇ by SIAM. Unauthorized reproduction of this article is prohibited. 
Proof. Here, we follow our convention and use the same notation for the difference operators in the interior and perpendicular to boundaries:

$$
\begin{aligned}
& \left(D^{x} \mathbf{q}_{i j k}\right)^{T} \mathbf{F}_{i j k}^{\mu}+\left(D^{y} \mathbf{q}_{i j k}\right)^{T} \mathbf{G}_{i j k}^{\mu}+\left(D^{z} \mathbf{q}_{i j k}\right)^{T} \mathbf{H}_{i j k}^{\mu} \\
& =\left({\stackrel{x}{T^{-1}}}_{i j k} D^{x} u_{i j k}+\stackrel{x}{u_{i j k}} D^{x} \frac{1}{T_{i j k}}\right) 1_{x}\left(\tau_{x x}\right)_{i j k} \\
& +\left(\stackrel{x}{x}^{-1}{ }_{i j k} D^{x} v_{i j k}+\stackrel{x}{v}_{i j k} D^{x} \frac{1}{T_{i j k}}\right) 1_{x}\left(\tau_{x y}\right)_{i j k} \\
& +\left(T^{-x}{ }_{i j k} D^{x} w_{i j k}+\stackrel{x}{w}_{i j k} D^{x} \frac{1}{T_{i j k}}\right) 1_{x}\left(\tau_{x z}\right)_{i j k} \\
& -D^{x} \frac{1}{T_{i j k}} 1_{x}\left(\stackrel{x}{u}_{i j k}\left(\tau_{x x}\right)_{i j k}+\stackrel{x}{v}_{i j k}\left(\tau_{x y}\right)_{i j k}+\stackrel{x}{w}_{i j k}\left(\tau_{x z}\right)_{i j k}\right) \\
& +\left({\stackrel{y}{T^{-1}}}_{i j k} D^{y} u_{i j k}+\stackrel{y}{u}_{i j k} D^{y} \frac{1}{T_{i j k}}\right) 1_{y}\left(\tau_{y x}\right)_{i j k} \\
& +\left({\stackrel{y}{T^{-1}}}^{i j k} D^{y} v_{i j k}+\stackrel{y}{v}_{i j k} D^{y} \frac{1}{T_{i j k}}\right) 1_{y}\left(\tau_{y y}\right)_{i j k} \\
& +\left(\stackrel{y}{T^{-1}}{ }_{i j k} D^{y} w_{i j k}+\stackrel{y}{w}_{i j k} D^{y} \frac{1}{T_{i j k}}\right) 1_{y}\left(\tau_{y z}\right)_{i j k} \\
& -D^{y} \frac{1}{T_{i j k}} 1_{y}\left(\stackrel{y}{u}_{i j k}\left(\tau_{x y}\right)_{i j k}+\stackrel{y}{v}_{i j k}\left(\tau_{y y}\right)_{i j k}+\stackrel{y}{w}_{i j k}\left(\tau_{y z}\right)_{i j k}\right) \\
& +\left({\stackrel{z}{T^{-1}}}_{i j k} D^{z} u_{i j k}+\stackrel{z}{u}_{i j k} D^{z} \frac{1}{T_{i j k}}\right) 1_{z}\left(\tau_{z x}\right)_{i j k}
\end{aligned}
$$

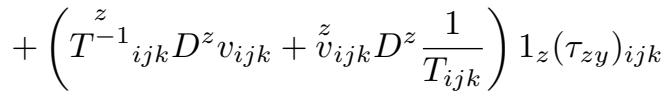

$$
\begin{aligned}
& +\left({\stackrel{z}{T^{-1}}}_{i j k} D^{z} w_{i j k}+\stackrel{z}{w}_{i j k} D^{z} \frac{1}{T_{i j k}}\right) 1_{z}\left(\tau_{z z}\right)_{i j k} \\
& -D^{z} \frac{1}{T_{i j k}} 1_{z}\left(\tilde{u}_{i j k}^{z}\left(\tau_{z x}\right)_{i j k}+\stackrel{z}{v}_{i j k}\left(\tau_{z y}\right)_{i j k}+\stackrel{z}{w}_{i j k}\left(\tau_{z z}\right)_{i j k}\right) .
\end{aligned}
$$

The terms from the energy equation cancel some terms form the momentum equations:

$$
\begin{array}{r}
\left(D^{x} \mathbf{q}_{i j k}\right)^{T} \mathbf{F}_{i j k}^{\mu}+\left(D^{y} \mathbf{q}_{i j k}\right)^{T} \mathbf{G}_{i j k}^{\mu}+\left(D^{z} \mathbf{q}_{i j k}\right)^{T} \mathbf{H}_{i j k}^{\mu} \\
=\left(T^{x}{ }_{i j k} D^{x} u_{i j k}\right) 1_{x}\left(\tau_{x x}\right)_{i j k}+\left(T^{-1}{ }_{i j k} D^{x} v_{i j k}\right) 1_{x}\left(\tau_{x y}\right)_{i j k} \\
+\left(T^{x}{ }_{i j k} D^{x} w_{i j k}\right) 1_{x}\left(\tau_{x z}\right)_{i j k} \\
+\left(T^{y}{ }_{i j k} D^{y} u_{i j k}\right) 1_{y}\left(\tau_{x y}\right)_{i j k}+\left(T^{y}{ }_{i j k} D^{y} v_{i j k}\right) 1_{y}\left(\tau_{y y}\right)_{i j k} \\
+\left(T^{y}{ }_{i j k} D^{y} w_{i j k}\right) 1_{y}\left(\tau_{z y}\right)_{i j k} \\
+\left(T^{z}{ }_{i j k} D^{z} u_{i j k}\right) 1_{z}\left(\tau_{x z}\right)_{i j k} \\
+\left(T^{z}{ }_{i j k} D^{z} v_{i j k}\right) 1_{z}\left(\tau_{y z}\right)_{i j k} \\
+\left(T^{z}{ }_{i j k} D^{z} w_{i j k}\right) 1_{z}\left(\tau_{z z}\right)_{i j k} .
\end{array}
$$

Copyright (c) by SIAM. Unauthorized reproduction of this article is prohibited. 
Using the definition of $1_{x, y, z}$ and factoring out $\mu / T_{i j k}$ leads to

$$
\begin{array}{r}
\left(D^{x} \mathbf{q}_{i j k}\right)^{T} \mathbf{F}_{i j k}^{\mu}+\left(D^{y} \mathbf{q}_{i j k}\right)^{T} \mathbf{G}_{i j k}^{\mu}+\left(D^{z} \mathbf{q}_{i j k}\right)^{T} \mathbf{H}_{i j k}^{\mu} \\
=\left(\frac{\mu}{T}\right)_{i j k}\left(\{\mathfrak{u}, \mathfrak{v}, \mathfrak{w}\}_{x, y, z}\right)_{i j k}
\end{array}
$$

where

$$
\begin{array}{r}
\left(\{\mathfrak{u}, \mathfrak{v}, \mathfrak{w}\}_{x, y, z}\right)_{i j k}=\frac{1}{\mu}\left(D^{x} u_{i j k}\left(\tau_{x x}\right)_{i j k}+D^{x} v_{i j k}\left(\tau_{x y}\right)_{i j k}+D^{x} w_{i j k}\left(\tau_{x z}\right)_{i j k}\right. \\
+D^{y} u_{i j k}\left(\tau_{y x}\right)_{i j k}+D^{y} v_{i j k}\left(\tau_{y y}\right)_{i j k}+D^{y} w_{i j k}\left(\tau_{y z}\right)_{i j k} \\
\left.+D^{z} u_{i j k}\left(\tau_{z x}\right)_{i j k}+D^{z} v_{i j k}\left(\tau_{z y}\right)_{i j k}+D^{z} w_{i j k}\left(\tau_{z z}\right)_{i j k}\right) \\
=\left(\mathfrak{u}_{x}\right)_{i j k}\left(\frac{4}{3}\left(\mathfrak{u}_{x}\right)_{i j k}-\frac{2}{3}\left(\left(\mathfrak{v}_{y}\right)_{i j k}+\left(\mathfrak{w}_{z}\right)_{i j k}\right)\right) \\
+\left(\mathfrak{v}_{x}\right)_{i j k}\left(\left(\mathfrak{u}_{y}\right)_{i j k}+\left(\mathfrak{v}_{x}\right)_{i j k}\right) \\
+\left(\mathfrak{w}_{x}\right)_{i j k}\left(\left(\mathfrak{u}_{z}\right)_{i j k}+\left(\mathfrak{w}_{x}\right)_{i j k}\right) \\
+\left(\mathfrak{u}_{y}\right)_{i j k}\left(\left(\mathfrak{u}_{y}\right)_{i j k}+\left(\mathfrak{v}_{x}\right)_{i j k}\right) \\
+\left(\mathfrak{v}_{y}\right)_{i j k}\left(\frac{4}{3}\left(\mathfrak{v}_{y}\right)_{i j k}-\frac{2}{3}\left(\left(\mathfrak{u}_{x}\right)_{i j k}+\left(\mathfrak{w}_{z}\right)_{i j k}\right)\right) \\
+\left(\mathfrak{w}_{y}\right)_{i j k}\left(\left(\mathfrak{v}_{z}\right)_{i j k}+\left(\mathfrak{w}_{y}\right)_{i j k}\right) \\
+\left(\mathfrak{u}_{z}\right)_{i j k}\left(\left(\mathfrak{u}_{z}\right)_{i j k}+\left(\mathfrak{w}_{x}\right)_{i j k}\right) \\
+\left(\mathfrak{v}_{z}\right)_{i j k}\left(\left(\mathfrak{v}_{z}\right)_{i j k}+\left(\mathfrak{w}_{y}\right)_{i j k}\right) \\
+\left(\mathfrak{w}_{z}\right)_{i j k}\left(\frac{4}{3}\left(\mathfrak{w}_{z}\right)_{i j k}-\frac{2}{3}\left(\left(\mathfrak{u}_{x}\right)_{i j k}+\left(\mathfrak{v}_{y}\right)_{i j k}\right)\right) .
\end{array}
$$

Since $(\mu / T)_{i j k}$ is nonnegative, it is sufficient to prove that $\left(\{\mathfrak{u}, \mathfrak{v}, \mathfrak{w}\}_{x, y, z}\right)_{i j k}$ is nonnegative. We suppress the common index $i j k$ and obtain

$$
\begin{aligned}
&\left(\{\mathfrak{u}, \mathfrak{v}, \mathfrak{w}\}_{x, y, z}\right)= \frac{4}{3}\left(\mathfrak{u}_{x}\right)^{2}-\frac{2}{3}\left(\mathfrak{v}_{y} \mathfrak{u}_{x}+\mathfrak{w}_{z} \mathfrak{u}_{x}\right)+\mathfrak{v}_{x} \mathfrak{u}_{y}+\mathfrak{v}_{x} \mathfrak{v}_{x}+\mathfrak{u}_{z} \mathfrak{w}_{x}+\mathfrak{w}_{x} \mathfrak{w}_{x} \\
&+ \mathfrak{u}_{y} \mathfrak{u}_{y}+\mathfrak{v}_{x} \mathfrak{u}_{y}+\frac{4}{3}\left(\mathfrak{v}_{y}\right)^{2}-\frac{2}{3}\left(\mathfrak{u}_{x} \mathfrak{v}_{y}+\mathfrak{w}_{z} \mathfrak{v}_{y}\right)+\mathfrak{v}_{z} \mathfrak{w}_{y}+\mathfrak{w}_{y} \mathfrak{w}_{y} \\
&+\mathfrak{u}_{z} \mathfrak{u}_{z}+\mathfrak{w}_{x} \mathfrak{u}_{z}+\mathfrak{v}_{z} \mathfrak{v}_{z}+\mathfrak{w}_{y} \mathfrak{v}_{z}+\frac{4}{3} \mathfrak{w}_{z}^{2}-\frac{2}{3}\left(\mathfrak{u}_{x} \mathfrak{w}_{z}+\mathfrak{v}_{y} \mathfrak{w}_{z}\right) \\
&=\frac{2}{3}\left(\left(\mathfrak{u}_{x}-\mathfrak{v}_{y}\right)^{2}+\left(\mathfrak{u}_{x}-\mathfrak{w}_{z}\right)^{2}+\left(\mathfrak{v}_{y}-\mathfrak{w}_{z}\right)^{2}\right) \\
&+\left(\mathfrak{v}_{x}+\mathfrak{u}_{y}\right)^{2}+\left(\mathfrak{u}_{z}+\mathfrak{w}_{x}\right)^{2}+\left(\mathfrak{v}_{z}+\mathfrak{w}_{y}\right)^{2} \geq 0 .
\end{aligned}
$$

In the derivation leading to (8), (1) was multiplied by $\mathbf{q}^{T}$ and integrated in space. The discrete counterpart will be to multiply (21) by $\mathbf{q}_{i j k}^{T}$ and sum over $\Omega_{N}$. To do this, we need the following notions.

Let $\left(u^{h}, v^{h}\right)=\sum_{i j k=0}^{N} h^{3} \mathcal{P}_{i j k} u_{i j k} v_{i j k}$ be an inner product where the superscript $h$ signifies that it is a field of values. $\mathcal{P}_{i j k}=\mathcal{P}_{i} \mathcal{P}_{j} \mathcal{P}_{k}$ are specific weights associated with the discrete norm. Specifically, $\mathcal{P}_{0}=\mathcal{P}_{N}=1 / 2$ and else $\mathcal{P}_{i}=1$. We will also use the product $\mathcal{P}_{j k}=\mathcal{P}_{j} \mathcal{P}_{k}$.

Furthermore, let $L^{2}\left(\Omega_{N}\right)$ denote the space of grid functions bounded in the norm, $\left\|u^{h}\right\|_{2}^{2}=\left(u_{h}, u_{h}\right)$. Furthermore, let $H^{1}\left(\Omega_{N}\right)$ denote the space where the variable and its differences (defined by $D^{x, y, z}$ ) are bounded in $L^{2}\left(\Omega_{N}\right)$.

Copyright (c) by SIAM. Unauthorized reproduction of this article is prohibited. 
With this definition of the $L^{2}$ space, the following SBP rule holds:

$$
\begin{array}{r}
\sum_{i j k=0}^{N} h^{3} \mathcal{P}_{i j k} \mathbf{q}_{i j k} D^{x} \mathbf{F}_{i j k} \\
(29)=-\sum_{j k=0}^{N} h^{2} \mathcal{P}_{j k} \mathbf{q}_{0 j k} \mathbf{F}_{0 j k}+\sum_{j k=0}^{N} h^{2} \mathcal{P}_{j k} \mathbf{q}_{N j k} \mathbf{F}_{N j k}-\sum_{i j k=0}^{N} h^{3} \mathcal{P}_{i j k}\left(D^{x} \mathbf{q}_{i j k}\right) \mathbf{F}_{i j k} .
\end{array}
$$

Remark. Note that $h^{3} \mathcal{P}_{i} \mathcal{P}_{j} \mathcal{P}_{k}$ is simply the measure of a volume element surrounding $x_{i}, y_{j}, z_{k}$. Similarly, $h^{2} \mathcal{P}_{j k}$ is the measure of a surface element at $x=0$ or $x=1$. Furthermore, all derivations can be straightforwardly generalized to the case with different grid sizes in different coordinate directions.

The next two lemmas further pave the way for the entropy estimate.

Lemma 3. Assume that $\rho_{i j k}, T_{i j k}>0$, that $\mathbf{f}_{i+1 / 2 j k}, \mathbf{g}_{i j+1 / 2 k}, \mathbf{h}_{i j k+1 / 2}$ are entropy conservative (or stable) numerical fluxes, and that the boundary fluxes are given as in (16) and (17). Then

$$
\sum_{i j k=0}^{N} h^{3} \mathcal{P}_{i j k} \mathbf{q}_{i j k}^{T}\left(D_{-}^{x} \mathbf{f}_{i+1 / 2 j k}+D_{-}^{y} \mathbf{g}_{i j+1 / 2 k}+D_{-}^{z} \mathbf{h}_{i j k+1 / 2}\right) \geq 0 .
$$

Proof. The scheme is equivalent to that in [PCN15], where this statement was proved.

Next, we need the geometric average, $\stackrel{g x}{T}_{i j k}=\sqrt{T_{i+1 j k} T_{i-1 j k}}$. Once again, we apply the convention to write the averaged direction with a capital index. At the boundary, we use $\stackrel{g x}{T}_{0 j k}=\sqrt{T_{1 j k} T_{0 j k}}$. (The obvious generalizations apply at the other boundaries.)

LEMma 4. Assume that $\rho_{i j k}, T_{i j k}>0$; then the following holds:

$$
\sum_{i j k=0}^{N} h^{3} \mathcal{P}_{i j k} \mathbf{q}_{i j k}^{T}\left(D^{x} \mathbf{F}^{\kappa}{ }_{i j k}+D^{y} \mathbf{G}^{\kappa}{ }_{i j k}+D^{z} \mathbf{H}^{\kappa}{ }_{i j k}+S A T_{i j k}^{\kappa}\right)=-D i f f_{\kappa},
$$

where

$$
=\sum_{i j k=0}^{N} h^{3} \mathcal{P}_{i j k} \frac{\kappa}{c_{v}}\left(\left(T_{i j k}\right)^{2}\left(D^{x} \frac{-1}{T_{i j k}}\right)^{2}+\left(T_{i j k}\right)^{2}\left(D^{y} \frac{-1}{T_{i j k}}\right)^{2}+\left(T_{i j k}^{g z}\right)^{2}\left(D^{z} \frac{-1}{T_{i j k}}\right)^{2}\right) .
$$

Proof. The proof follows from a straightforward application of the SBP rule (29) and (27).

Lemma 5. If $T_{i j k}>0$, then

$$
\begin{array}{r}
\sum_{i j k=0}^{N} h^{3} \mathcal{P}_{i j k} \mathbf{q}_{i j k}^{T}\left(D^{x} \mathbf{F}^{\mu}{ }_{i j k}+D^{y} \mathbf{G}^{\mu}{ }_{i j k}+D^{z} \mathbf{H}^{\mu}{ }_{i j k}\right) \\
=\sum_{i j k=0}^{N} h^{3} \mathcal{P}_{i j k} \frac{\mu}{T_{i j k}}\left(\{\mathfrak{u}, \mathfrak{v}, \mathfrak{w}\}_{x, y, z}\right)_{i j k},
\end{array}
$$

Copyright $@$ by SIAM. Unauthorized reproduction of this article is prohibited. 
and

$$
\sum_{i j k=0}^{N} h^{3} \mathcal{P}_{i j k} \mathbf{q}_{i j k}^{T} S A T_{i j k}^{\mu} \leq 0
$$

Proof. The first result is a direct consequence of Lemma 2. The second follows immediately from (5) and (22).

Note that the first statement in the lemma does not involve any boundary conditions, since the boundary terms $\mathbf{q}_{0 j k} F_{0 j k}^{\mu}=0$. This is a consequence of the specific boundary averages (19) and is consistent with the continuous counterpart. (See (7), where the velocity part vanishes.)

We are finally ready to derive the semidiscrete equivalent of (8).

Proposition 6. Assume that the initial state of the solution variables, $\mathbf{u}^{h}(0)$, and the temperature, $T^{h}(0)$, are bounded in $L^{2}\left(\Omega_{N}\right)$. Furthermore, assume that $T^{h}(t), \rho^{h}(t) \geq \epsilon>0, t \in[0, \mathcal{T}]$. Then the scheme (21) is entropy stable and its solutions satisfy $\mathbf{u}^{h} \in C\left(0, \mathcal{T} ;\left(L^{2}\left(\Omega_{N}\right)\right)^{5}\right)$ and $p^{h},\left(\rho\left(u^{2}+v^{2}+w^{2}\right)\right)^{h} \in C\left(0, \mathcal{T} ; L^{2}\left(\Omega_{N}\right)\right)$. Furthermore, $\log \left(T^{h}\right) \in L^{2}\left(0, \mathcal{T} ; H^{1}\left(\Omega_{N}\right)\right)$.

Proof. Once again we remind that we consider only the boundary terms at $x=0$; all other terms are dropped. Multiplying the scheme (21) by $h^{3} p_{i j k} q_{i j k}^{T}$ and summing in space leads to

$$
\begin{array}{r}
\sum_{i, j, k=0}^{N} h^{3} \mathcal{P}_{i j k}\left(U_{i j k}\right)_{t}+\sum_{i, j, k=0}^{N} h^{3} \mathbf{q}_{i j k}^{T} \mathcal{P}_{i j k}\left(D_{-}^{x} \mathbf{f}_{i+1 / 2 j k}+D_{-}^{y} \mathbf{g}_{i j+1 / 2 k}+D_{-}^{z} \mathbf{h}_{i, j, k+1 / 2}\right) \\
=\sum_{i, j, k=0}^{N} h^{3} \mathbf{q}_{i j k}^{T} \mathcal{P}_{i j k}\left(D^{x} \mathbf{F}_{i j k}+D^{y} \mathbf{G}_{i j k}+D^{z} \mathbf{H}_{i j k}\right)+\sum_{i, j, k=0}^{N} h^{3} \mathbf{q}_{i j k} \mathcal{P}_{i j k} S A T_{i j k} .
\end{array}
$$

Sum by parts and use Lemmas 3,4 , and 5 to obtain

$$
\sum_{i, j, k=0}^{N} h^{3}\left(U_{i j k}\right)_{t}+\sum_{i j k} h^{3} \mathcal{P}_{i j k} \frac{\mu}{T_{i j k}}\left(\{\mathfrak{u}, \mathfrak{v}, \mathfrak{w}\}_{x, y, z}\right)_{i j k}+D i f f_{\kappa} \leq 0 .
$$

From (31), we have

$$
\sum_{i, j, k=0}^{N} h^{3}\left(U_{i j k}\right)_{t} \leq 0
$$

since the second sum and Diff $\kappa_{\kappa}$ are positive. We use a standard argument to obtain an $L^{2}$ bound on the variables. We repeat the calculation for the entropy $\bar{U}=U-$ $U\left(\mathbf{u}_{0}\right)-U^{\prime}\left(\mathbf{u}_{0}\right)^{T}\left(\mathbf{u}-\mathbf{u}_{0}\right)$, where $\mathbf{u}_{0}$ is a constant state. (This is an affine change, which ensures that $\bar{U}$ is an entropy.) We choose the constant state as $\mathbf{u}_{0}^{1}=\rho_{0} \geq c>0$, $\mathbf{u}_{0}^{2,3,4}=0$, and $\mathbf{u}_{0}^{5}=E_{0} \geq c>0$. ( $c$ is a constant.) This corresponds to a state at rest with constant density, temperature, and pressure. 
The entropy $\bar{U}$ satisfies the analog estimate (31). We can recast this as

$$
\begin{array}{r}
\frac{1}{2} \sum_{i, j, k=0}^{N}\left\{h^{3} \mathcal{P}_{i j k}\left(\mathbf{u}-\mathbf{u}_{0}\right)^{T} U^{\prime \prime}(\theta(\mathcal{T}))\left(\mathbf{u}-\mathbf{u}_{0}\right)\right\}_{i j k} \\
+\int_{0}^{\mathcal{T}} \sum_{i, j, k=0}^{N} h^{3} \mathcal{P}_{i j k}\left(\left(D^{x} \mathbf{q}_{i j k}\right)^{T} \mathbf{F}_{i j k}+\left(D^{y} \mathbf{q}_{i j k}\right)^{T} \mathbf{G}_{i j k}\right. \\
\left.+\left(D^{z} \mathbf{q}_{i j k}\right)^{T} \mathbf{H}_{i j k}-\mathbf{q}_{i j k} S A T_{i j k}\right) d t \\
\leq \sum_{i, j, k=0}^{N} h^{3} \bar{U}\left(\mathbf{u}^{h}(0)\right),
\end{array}
$$

where $\theta(t)$ is an average state between $\mathbf{u}$ and $\mathbf{u}_{0}$. Since $\rho_{i j k}, T_{i j k} \geq \epsilon>0$ and $\rho_{0}, E_{0}>$ $c$, the density and temperature in $\theta(t)$ are positive and bounded away from 0 . Hence, and since $U$ is convex, $U^{\prime \prime}(\theta(t))$, for $t \in[0, \mathcal{T}]$, is symmetric positive definite (with all eigenvalues bounded away from 0). (See [Har83, eq. 2.8a].) Consequently, we obtain an $L^{2}$ bound on $\mathbf{u}=\left(\rho^{h},(\rho u)^{h},(\rho v)^{h},(\rho w)^{h}, E^{h}\right)^{T}$ (continuously in time). (These ideas were outlined in [Daf10, Chapter 5] and also presented in detail in [Svä15]. The derivation uses a multivariate version of Taylor's theorem with a Lagrange remainder. See [Tre13, pp. 350].)

We obtain from estimate (32) and Lemma 4

$$
\begin{aligned}
\int_{0}^{T} \sum_{i j k=0}^{N} h^{3} \mathcal{P}_{i j k}\left(\left(T_{i j k}\right)^{2}\left(D^{x} \frac{-1}{T_{i j k}}\right)^{2}\right. & +\left({ }^{g y}\right. \\
T_{i j k} & )^{2}\left(D^{y} \frac{-1}{T_{i j k}}\right)^{2} \\
& \left.+\left(\stackrel{g}{T}_{i j k}\right)^{2}\left(D^{z} \frac{-1}{T_{i j k}}\right)^{2}\right) d t \leq \mathcal{C} .
\end{aligned}
$$

For positive quantities, $\xi_{1}, \xi_{2}>0$, the $\log$ average is $\stackrel{l x}{\xi}=\left(\xi_{1}-\xi_{2}\right) /\left(\log \left(\xi_{1}\right)-\log \left(x_{2}\right)\right)$. For the same quantities, the geometric average is $\stackrel{g x}{\xi}=\sqrt{\xi_{1} \xi_{2}}$, and it holds that $\stackrel{g x}{\xi} \leq \stackrel{l x}{\xi}$ [Car66]. Since

$$
D^{x} \log \left(T_{i}\right)=\frac{1}{T_{i j k}} D^{x} T_{i}=\frac{1}{T_{i j k}}\left(T_{i j k}^{g x}\right)^{2} D^{x} \frac{-1}{T_{i}}
$$

and $\left.\stackrel{g x}{\left(T_{i j k}\right.}\right)^{2} / \stackrel{l x}{T}_{i j k} \leq \stackrel{g x}{T}_{i j k}$, the bound (33) implies an estimate of $D^{x} \log \left(T^{h}\right)$. By the Poincaré inequality (noting that $T^{h}$ has to be bounded and nonzero on a nonvanishing subset), we obtain $\log \left(T^{h}\right) \in L^{2}\left(0, \mathcal{T} ; L^{2}\left(\Omega_{N}\right)\right.$ ). (See [FV10, Lemma 2.1] for a statement of the Poincaré inequality, and [Svä16, Theorem B.1] for the discrete counterpart.) Therefore, $T^{h}>0$ a.e. as $h \rightarrow 0$. (Note that $T^{h}>0$ implies that we only need to a priori assume that $\rho^{h} \geq 0$.)

Finally, from the assumption that $\rho^{h}>0$, we obtain from (32) that $E_{h} \in L^{2}\left(\Omega_{N}\right)$. Furthermore, $\rho^{h}>0$ and the previously derived result, $T^{h}>0$, implies that $p^{h}>0$. Since $E_{h}=\frac{p_{h}}{\gamma-1}+\frac{1}{2}\left(\rho\left(u^{2}+v^{2}+w^{2}\right)^{h}\right)$, we conclude that $p_{h}$ and $\left(\rho\left(u^{2}+v^{2}+w^{2}\right)^{h}\right)(>0)$ are also bounded in $L^{2}$.

Up until now, the analysis is essentially analogous to [PCN15]. We point out a few differences. The authors use the entropy variables to discretize the diffusive 
fluxes, which makes the generalization to high-order schemes immediate. Here, we discretize the diffusive flux with the conservative variables. The reason for this is to obtain a bound on the velocity gradients.

We continue with a lemma that is the discrete equivalent of (13).

LEMmA 7. The following SBP rule holds when all boundaries but $i=0$ are ignored:

$$
\sum_{i j k=0}^{N} h^{3}\left(\mathfrak{u}_{x}\right)_{i j k} \mathcal{P}_{i j k}\left(\mathfrak{v}_{y}\right)_{i j k}=-\sum_{j k=0}^{N} h^{2} u_{0 j k} \mathcal{P}_{i j k}\left(\mathfrak{v}_{y}\right)_{0 j k}+\sum_{i j k=0}^{N} h^{3}\left(\mathfrak{u}_{y}\right)_{i j k} \mathcal{P}_{i j k}\left(\mathfrak{v}_{x}\right)_{i j k}
$$

Proof. Use the SBP rule (29) to obtain

$$
\begin{array}{r}
\sum_{i j k=0}^{N} \mathcal{P}_{i j k} h^{3}\left(\mathfrak{u}_{x}\right)_{i j k}\left(\mathfrak{v}_{y}\right)_{i j k}=\sum_{i j k=0}^{N} \mathcal{P}_{i j k} h^{3}\left(D^{x} u\right)_{i j k}\left(D^{y} v\right)_{i j k} \\
=-\sum_{j k=0}^{N} h^{2} \mathcal{P}_{j k}\left(u_{0 j k}\left(D^{y} v\right)_{0 j k}+u_{N j k}\left(D^{y} v\right)_{N j k}\right)-\sum_{i j k=0}^{N} h^{3} \mathcal{P}_{i j k} u_{i j k}\left(D^{x} D^{y} v\right)_{i j k} .
\end{array}
$$

Furthermore, since $D^{x}$ and $D^{y}$ commute, we can apply (29) once more:

$$
\begin{array}{r}
\sum_{i j k=0}^{N} h^{3}\left(\mathfrak{u}_{x}\right)_{i j k} \mathcal{P}_{i j k}\left(\mathfrak{v}_{y}\right)_{i j k} \\
=-\sum_{j k=0}^{N} h^{2} \mathcal{P}_{j k}\left(u_{0 j k}\left(D^{y} v\right)_{0 j k}+u_{N j k}\left(D^{y} v\right)_{N j k}\right) \\
+\sum_{i k=0}^{N} h^{2} \mathcal{P}_{i k}\left(u_{i 0 k}\left(D^{x} v\right)_{i 0 k}-u_{i N k}\left(D^{x} v\right)_{i N k}\right)+\sum_{i j k=0}^{N} h^{3}\left(D^{y} u\right)_{i j k} \mathcal{P}_{i j k}\left(D^{x} v\right)_{i j k} .
\end{array}
$$

Keeping the boundary terms at $x=0$, we arrive at (34).

Now we introduce $\mathbf{v}^{h}$, which denotes the velocity vector field, $\left\{\mathbf{v}^{h}\right\}_{i j k}=(u, v, w)_{i j k}$. We have come to the main result of this paper, namely, the derivation of a bound on the velocity gradients, which in turn requires the full no-slip condition.

TheOrem 8. Under the assumptions of Proposition 6 and if $T_{i j k} \leq T_{\max } \in$ $L^{\infty}\left(0, \mathcal{T}, L^{\infty}\left(\Omega_{N}\right)\right)$, then $\mathbf{v}^{h} \in L^{2}\left(0, \mathcal{T},\left(H^{1}\left(\Omega_{N}\right)\right)^{3}\right)$.

Proof. From the entropy estimate $(31)$, we extract the $\left(\{\mathfrak{u}, \mathfrak{v}, \mathfrak{w}\}_{x, y, z}\right)$ terms and $S A T^{\mu}$, which are independently bounded. We have

$$
\int_{0}^{\mathcal{T}} \sum_{i, j, k=0}^{N} h^{3} \mathcal{P}_{i j k}\left(\left(\frac{\mu}{T}\right)_{i j k}\left(\{\mathfrak{u}, \mathfrak{v}, \mathfrak{w}\}_{x, y, z}\right)_{i j k}-\mathbf{q}_{i j k} S A T_{i j k}^{\mu}\right) d t \leq \mathcal{C} .
$$

Using the assumption $T_{i j k} \in L^{\infty}$, the previous expression bounds

$$
\begin{aligned}
\mathcal{C} & \geq \int_{0}^{\mathcal{T}} \sum_{i, j, k=0}^{N} h^{3} \mathcal{P}_{i j k}\left(\frac{\mu}{T}\right)_{i j k}\left(\left(\{\mathfrak{u}, \mathfrak{v}, \mathfrak{w}\}_{x, y, z}\right)_{i j k}-\mathbf{q}_{i j k} S A T_{i j k}^{n o-s l i p}\right) d t \\
& \geq \frac{\mu}{T_{\max }} \int_{0}^{\mathcal{T}} \sum_{i, j, k=0}^{N} h^{3} \mathcal{P}_{i j k}\left(\left(\{\mathfrak{u}, \mathfrak{v}, \mathfrak{w}\}_{x, y, z}\right)_{i j k}-\mathbf{q}_{i j k} S A T_{i j k}^{n o-s l i p}\right) d t .
\end{aligned}
$$

Copyright (c) by SIAM. Unauthorized reproduction of this article is prohibited. 
Once again, we drop the common indices and rewrite the velocity differences:

$$
\begin{array}{r}
\left(\{\mathfrak{u}, \mathfrak{v}, \mathfrak{w}\}_{x, y, z}\right) \\
=\frac{2}{3}\left(\left(\mathfrak{u}_{x}-\mathfrak{v}_{y}\right)^{2}+\left(\mathfrak{u}_{x}-\mathfrak{w}_{z}\right)^{2}+\left(\mathfrak{v}_{y}-\mathfrak{w}_{z}\right)^{2}\right) \\
+\left(\mathfrak{v}_{x}+\mathfrak{u}_{y}\right)^{2}+\left(\mathfrak{u}_{z}+\mathfrak{w}_{x}\right)^{2}+\left(\mathfrak{v}_{z}+\mathfrak{w}_{y}\right)^{2} \\
=\frac{2}{3}\left(\left(\mathfrak{u}_{x}^{2}+\mathfrak{v}_{y}^{2}-2 \mathfrak{u}_{x} \mathfrak{v}_{y}\right)+\left(\mathfrak{u}_{x}^{2}+\mathfrak{w}_{z}^{2}-2 \mathfrak{u}_{x} \mathfrak{w}_{z}\right)+\left(\mathfrak{v}_{y}^{2}+\mathfrak{w}_{z}^{2}-2 \mathfrak{v}_{y} \mathfrak{w}_{z}\right)\right) \\
+\frac{1}{3}\left(\mathfrak{v}_{x}+\mathfrak{u}_{y}\right)^{2}+\frac{2}{3}\left(\mathfrak{v}_{x}^{2}+\mathfrak{u}_{y}^{2}+2 \mathfrak{v}_{x} \mathfrak{u}_{y}\right) \\
+\frac{1}{3}\left(\mathfrak{u}_{z}+\mathfrak{w}_{x}\right)^{2}+\frac{2}{3}\left(\mathfrak{u}_{z}^{2}+\mathfrak{w}_{x}^{2}+2 \mathfrak{u}_{z} \mathfrak{w}_{x}\right) \\
+\frac{1}{3}\left(\mathfrak{v}_{z}+\mathfrak{w}_{y}\right)^{2}+\frac{2}{3}\left(\mathfrak{v}_{z}^{2}+\mathfrak{w}_{y}^{2}+2 \mathfrak{v}_{z} \mathfrak{w}_{y}\right) \\
=\frac{1}{3}\left(\mathfrak{v}_{x}+\mathfrak{u}_{y}\right)^{2}+\frac{2}{3}\left(\mathfrak{v}_{x}^{2}+\mathfrak{u}_{x}^{2}+\mathfrak{u}_{y}^{2}+\mathfrak{v}_{y}^{2}+2\left(\mathfrak{v}_{x} \mathfrak{u}_{y}-\mathfrak{u}_{x} \mathfrak{v}_{y}\right)\right) \\
+\frac{1}{3}\left(\mathfrak{u}_{z}+\mathfrak{w}_{x}\right)^{2}+\frac{2}{3}\left(\mathfrak{u}_{z}^{2}+\mathfrak{w}_{z}^{2}+\mathfrak{w}_{x}^{2}+\mathfrak{u}_{x}^{2}+2\left(\mathfrak{u}_{z} \mathfrak{w}_{x}-\mathfrak{u}_{x} \mathfrak{w}_{z}\right)\right) \\
+\frac{1}{3}\left(\mathfrak{v}_{z}+\mathfrak{w}_{y}\right)^{2}+\frac{2}{3}\left(\mathfrak{v}_{z}^{2}+\mathfrak{w}_{z}^{2}+\mathfrak{w}_{y}^{2}+\mathfrak{v}_{y}^{2}+2\left(\mathfrak{v}_{z} \mathfrak{w}_{y}-\mathfrak{v}_{y} \mathfrak{w}_{z}\right)\right) \geq 0 .
\end{array}
$$

Then we use this expression in (35):

$$
\begin{array}{r}
\sum_{i j k=0}^{N} p_{i j k} h^{3}\left(\{\mathfrak{u}, \mathfrak{v}, \mathfrak{w}\}_{x, y, z}-\mathbf{q}_{i j k} S A T^{n o-s l i p}\right) \\
=\sum_{i j k=0}^{N} p_{i j k} h^{3}\left(\frac{1}{3}\left(\mathfrak{v}_{x}+\mathfrak{u}_{y}\right)^{2}+\frac{2}{3}\left(\mathfrak{v}_{x}^{2}+\mathfrak{u}_{x}^{2}+\mathfrak{u}_{y}^{2}+\mathfrak{v}_{y}^{2}+2\left(\mathfrak{v}_{x} \mathfrak{u}_{y}-\mathfrak{u}_{x} \mathfrak{v}_{y}\right)\right)\right. \\
+\frac{1}{3}\left(\mathfrak{u}_{z}+\mathfrak{w}_{x}\right)^{2}+\frac{2}{3}\left(\mathfrak{u}_{z}^{2}+\mathfrak{w}_{z}^{2}+\mathfrak{w}_{x}^{2}+\mathfrak{u}_{x}^{2}+2\left(\mathfrak{u}_{z} \mathfrak{w}_{x}-\mathfrak{u}_{x} \mathfrak{w}_{z}\right)\right) \\
+\frac{1}{3}\left(\mathfrak{v}_{z}+\mathfrak{w}_{y}\right)^{2}+\frac{2}{3}\left(\mathfrak{v}_{z}^{2}+\mathfrak{w}_{z}^{2}+\mathfrak{w}_{y}^{2}+\mathfrak{v}_{y}^{2}+2\left(\mathfrak{v}_{z} \mathfrak{w}_{y}-\mathfrak{v}_{y} \mathfrak{w}_{z}\right)\right) \\
\left.-\mathbf{q}_{i j k} S A T_{i j k}^{\text {no-slip }}\right) .
\end{array}
$$

We use Lemma 7 repeatedly to rewrite the indefinite terms. We show the technique for one term (keeping only the boundary terms at $x=0$ ):

$$
\begin{array}{r}
\sum_{i j k=0}^{N} \mathcal{P}_{i j k} h^{3} \frac{4}{3}\left(\mathfrak{v}_{x} \mathfrak{u}_{y}-\mathfrak{u}_{x} \mathfrak{v}_{y}\right)_{i j k} \\
=\sum_{i j k=0}^{N} \mathcal{P}_{i j k} h^{3} \frac{4}{3}\left(\mathfrak{v}_{x} \mathfrak{u}_{y}-\mathfrak{u}_{y} \mathfrak{v}_{x}\right)_{i j k}+\sum_{j k=0}^{N} \mathcal{P}_{j k} h^{2} \frac{4}{3} u_{0 j k}\left(\mathfrak{v}_{y}\right)_{0 j k} .
\end{array}
$$

Or, we could equally well have manipulated the other term:

$$
\begin{array}{r}
\sum_{i j k=0}^{N} \mathcal{P}_{i j k} h^{3} \frac{4}{3}\left(\mathfrak{v}_{x} \mathfrak{u}_{y}-\mathfrak{u}_{x} \mathfrak{v}_{y}\right)_{i j k} \\
=\sum_{i j k=0}^{N} \mathcal{P}_{i j k} h^{3} \frac{4}{3}\left(\mathfrak{v}_{y} \mathfrak{u}_{x}-\mathfrak{u}_{x} \mathfrak{v}_{y}\right)_{i j k}-\sum_{j k=0}^{N} \mathcal{P}_{j k} h^{2} \frac{4}{3}\left(v_{0 j k}\left(\mathfrak{u}_{y}\right)_{0 j k}\right) .
\end{array}
$$

Copyright (c) by SIAM. Unauthorized reproduction of this article is prohibited. 
In order for all boundaries to get a symmetric contribution of boundary terms, we will take the average of the two:

$$
\sum_{i j k=0}^{N} \mathcal{P}_{i j k} h^{3} \frac{4}{3}\left(\mathfrak{v}_{x} \mathfrak{u}_{y}-\mathfrak{u}_{x} \mathfrak{v}_{y}\right)_{i j k}=\frac{1}{2} \sum_{j k=0}^{N} \mathcal{P}_{j k} h^{2} \frac{4}{3}\left(-v_{0 j k}\left(\mathfrak{u}_{y}\right)_{0 j k}+u_{0 j k}\left(\mathfrak{v}_{y}\right)_{0 j k}\right) .
$$

Similarly, and by keeping only the $x=0$ terms,

$$
\sum_{i j k=0}^{N} \mathcal{P}_{i j k} h^{3} \frac{4}{3}\left(\mathfrak{u}_{z} \mathfrak{w}_{x}-\mathfrak{u}_{x} \mathfrak{w}_{z}\right)_{i j k}=\frac{1}{2} \sum_{j k=0}^{N} \mathcal{P}_{j k} h^{2} \frac{4}{3}\left(u_{0 j k}\left(\mathfrak{w}_{z}\right)_{0 j k}-w_{0 j k}\left(\mathfrak{u}_{z}\right)_{0 j k}\right)
$$

and

$$
\sum_{i j k=0}^{N} \mathcal{P}_{i j k} h^{3} \frac{4}{3}\left(\mathfrak{v}_{z} \mathfrak{w}_{y}-\mathfrak{v}_{y} \mathfrak{w}_{z}\right)_{i j k}=0
$$

Using the above technique, we remove the indefinite terms in the domain and replace them with indefinite boundary terms. These in turn are possible to bound using $S A T^{\mu}$. We have

$$
\begin{array}{r}
\sum_{i j k=0}^{N} \mathcal{P}_{i j k} h^{3}\left(\{\mathfrak{u}, \mathfrak{v}, \mathfrak{w}\}_{x, y, z}-\mathbf{q}_{i j k} S A T^{n o-s l i p}\right) \\
=\sum_{i j k=0}^{N} \mathcal{P}_{i j k} h^{3}\left(\frac{1}{3}\left(\mathfrak{v}_{x}+\mathfrak{u}_{y}\right)^{2}+\frac{2}{3}\left(\mathfrak{v}_{x}^{2}+\mathfrak{u}_{y}^{2}+\mathfrak{u}_{x}^{2}+\mathfrak{v}_{y}^{2}\right)\right. \\
+\frac{1}{3}\left(\mathfrak{u}_{z}+\mathfrak{w}_{x}\right)^{2}+\frac{2}{3}\left(\mathfrak{u}_{z}^{2}+\mathfrak{w}_{x}^{2}+\mathfrak{u}_{x}^{2}+\mathfrak{w}_{z}^{2}\right) \\
+\frac{1}{3}\left(\mathfrak{v}_{z}+\mathfrak{w}_{y}\right)^{2}+\frac{2}{3}\left(\mathfrak{v}_{z}^{2}+\mathfrak{w}_{y}^{2}+\mathfrak{v}_{y}^{2}+\mathfrak{w}_{z}^{2}\right) \\
-\sum_{j k=0}^{N} h^{2} \mathcal{P}_{j k} \mathbf{q}_{0 j k} S A T_{0 j k}^{n o-s l i p} \\
-\sum_{j k=0}^{N} h^{2} \mathcal{P}_{j k} \frac{2}{3}\left(v_{0 j k}\left(\mathfrak{u}_{y}\right)_{0 j k}-u_{0 j k}\left(\mathfrak{v}_{y}\right)_{0 j k}-u_{0 j k}\left(\mathfrak{w}_{z}\right)_{0 j k}+w_{0 j k}\left(\mathfrak{u}_{z}\right)_{0 j k}\right) .
\end{array}
$$

From $\mathbf{q}_{0 j k} S A T_{0 j k}^{\text {no-slip }}$ we will get quadratic velocity terms. However, the indefinite boundary terms are products of a velocity and a velocity derivative. We need quadratic velocity derivative terms on the boundary in order to bound the indefinite terms. Such terms can be extracted from the sum. We introduce

$$
\begin{array}{r}
D i f f \\
\sum_{i j k=0}^{N} \mathcal{P}_{i j k} h^{3} \frac{1}{3}\left(\left(\mathfrak{v}_{x}+\mathfrak{u}_{y}\right)^{2}+\left(\mathfrak{u}_{z}+\mathfrak{w}_{x}\right)^{2}+\left(\mathfrak{v}_{z}+\mathfrak{w}_{y}\right)^{2}\right)_{i j k} \\
+\sum_{i j k=1}^{N-1} \mathcal{P}_{i j k} h^{3} \frac{2}{3}\left(\mathfrak{v}_{x}^{2}+\mathfrak{u}_{y}^{2}+\mathfrak{u}_{x}^{2}+\mathfrak{v}_{y}^{2}+\mathfrak{u}_{z}^{2}+\mathfrak{w}_{x}^{2}+\mathfrak{u}_{x}^{2}+\mathfrak{w}_{z}^{2}+\mathfrak{v}_{z}^{2}+\mathfrak{w}_{y}^{2}+\mathfrak{v}_{y}^{2}+\mathfrak{w}_{z}^{2}\right)_{i j k}
\end{array}
$$

Copyright (c) by SIAM. Unauthorized reproduction of this article is prohibited. 
and

$$
=\sum_{j k=0}^{N} \mathcal{P}_{0 j k} h^{3}\left(\mathfrak{v}_{x}^{2}+\mathfrak{u}_{y}^{2}+\mathfrak{u}_{x}^{2}+\mathfrak{v}_{y}^{2}+\mathfrak{u}_{z}^{2}+\mathfrak{w}_{x}^{2}+\mathfrak{u}_{x}^{2}+\mathfrak{w}_{z}^{2}+\mathfrak{v}_{z}^{2}+\mathfrak{w}_{y}^{2}+\mathfrak{v}_{y}^{2}+\mathfrak{w}_{z}^{2}\right)_{0 j k} .
$$

Furthermore, we denote the analogous terms on the remaining boundaries as $B T_{x=1, y z}$. Using the fact that $p_{0 j k}=1 / 2$ and $\mathbf{q}_{0 j k} S A T_{0 j k}^{n o-s l i p}=\sigma\left(u^{2}+v^{2}+w^{2}\right)_{0 j k}$, we rewrite (36) as

$$
\begin{array}{r}
\sum_{i j k} \mathcal{P}_{i j k} h^{3}\left(\{\mathfrak{u}, \mathfrak{v}, \mathfrak{w}\}_{x, y, z}-\mathbf{q}_{i j k} S A T^{n o-s l i p}\right) \\
=D_{i f f_{\mu}}+\frac{1}{3}\left(B T_{x=0}+B T_{x=1, y z}\right)-\sum_{j k=0}^{N} h^{2} \mathcal{P}_{j k} \sigma\left(u^{2}+v^{2}+w^{2}\right)_{0 j k} \\
-\sum_{j k=0}^{N} h^{2} \mathcal{P}_{j k} \frac{2}{3}\left(v_{0 j k}\left(\mathfrak{u}_{y}\right)_{0 j k}-u_{0 j k}\left(\mathfrak{v}_{y}\right)_{0 j k}-u_{0 j k}\left(\mathfrak{w}_{z}\right)_{0 j k}+w_{0 j k}\left(\mathfrak{u}_{z}\right)_{0 j k}\right) .
\end{array}
$$

With $\sigma \leq-\frac{1}{3 p_{0} h}$ and Proof 8 , it is straightforward to complete the squares and conclude that

$$
\begin{array}{r}
\mathbf{B T}_{\mathbf{x}=\mathbf{0}}=B T_{x=0}-\sum_{j k} h^{2} \mathcal{P}_{j k} \sigma\left(u^{2}+v^{2}+w^{2}\right) \\
-\sum_{j k} h^{2} \mathcal{P}_{j k} \frac{2}{3}\left(v_{0 j k}\left(\mathfrak{u}_{y}\right)_{0 j k}+u_{0 j k}\left(\mathfrak{v}_{y}\right)_{0 j k}-u_{0 j k}\left(\mathfrak{w}_{z}\right)+w_{0 j k}\left(\mathfrak{u}_{z}\right)_{0 j k}\right) \geq 0 .
\end{array}
$$

Tracing our steps back to (35), we see that

$$
\begin{array}{r}
\mathcal{C} \geq \frac{\mu}{T_{\max }} \int_{0}^{\mathcal{T}} \sum_{i, j, k=0}^{N} h^{3} \mathcal{P}_{i j k}( \\
\left.\left(\{\mathfrak{u}, \mathfrak{v}, \mathfrak{w}\}_{x, y, z}\right)_{i j k}-\mathbf{q}_{i j k} S A T_{i j k}^{\text {no-slip }}\right) d t \\
=\frac{\mu}{T_{\max }} \int_{0}^{\mathcal{T}}\left(D_{i f f_{\mu}}+\mathbf{B T}_{\mathbf{x}=\mathbf{0}}+\mathbf{R}\right) d t .
\end{array}
$$

We have shown that $\mathbf{B} \mathbf{T}_{\mathbf{x}=\mathbf{0}} \geq 0$ and $\mathbf{R} \geq 0$ represent the corresponding expressions for all the other boundaries. Hence, we obtain a bound on Diff $\mu$, i.e., all velocity derivatives.

Finally, the $L^{2}$ bound on $\mathbf{v}$ follows by the following argument. Since $\rho>0$ is conserved, there is a nonvanishing subset, $\mathcal{B}$, where $\rho \geq$ constant $>0$. Since $\rho \mathbf{v} \in L^{2}$, we have $\mathbf{v} \in L^{2}(\mathcal{B})$. By the Poincaré inequality, we obtain $\mathbf{v} \in L^{2}\left(\Omega_{N}\right)$. (See [FV10, Svä16].)

4. Conclusions. We have proposed an entropy stable finite difference scheme for the Navier-Stokes equations. The main difference from standard entropy stable formulations is that the diffusive terms are discretized in the standard variables rather than entropy variables. This was necessary to prove a bound on velocity gradients, which in turn is a necessary step towards well-posedness. The bound on the velocity gradients required a precise discrete formulation of the no-slip boundary conditions.

There are two properties that are essential for the analysis in this paper. The first is that the difference operators should have a summation-by-parts (SBP) property. 
(For Galerkin-type schemes this would be met by exact integration.) The second is a discrete Leibniz rule which was used in (25). Any SBP operator that simultaneously satisfies a Leibniz rule can be used to construct equivalent schemes for the diffusive terms by replacing the current averages of the fluxes and the difference operators with the corresponding ones for the new operators. (For the convective terms there are already high-order entropy stable schemes available.)

\section{REFERENCES}

[Car66] B.C. Carlson, Some inequalities for hypergeometric functions, Proc. Amer. Math. Soc., 17 (1966), pp. 32-39.

$\left[\mathrm{CFN}^{+} 16\right]$ M.H. Carpenter, T.C. Fisher, E.J. Nielsen, M. Parsani, M. Svärd, and N. YaMALEEV, Entropy stable summation-by-parts formulations for compressible computational fluid dynamics, in Handbook of Numerical Methods for Hyperbolic Problems, Handb. Numer. Anal. 17, R. Abgrall and C.-W. Shu, eds., Elsevier/North-Holland, Amsterdam, 2016, pp. 495-524.

[Daf10] C.M. Dafermos, Hyperbolic Conservation Laws in Continuum Physics, 3rd ed., Springer-Verlag, Berlin, Heidelberg, 2010.

[FC13] T.C. Fisher AND M.H. CARPENTER, High-order entropy stable finite difference schemes for nonlinear conservation laws: Finite domains, J. Comput. Phys., 252 (2013), pp. $518-557$.

[FV10] E. FeIreisl AND A. VAsSEur, New perspectives in fluid dynamics: Mathematical analysis of a model proposed by Howard Brenner, in New Directions in Mathematical Fluid Mechanics: The Alexander V. Kazhikhov Memorial Volume, Adv. Math. Fluid Mech., Andrei V. Fursikov, Giovanni P. Galdi, and Vladislav V. Pukhnachev, eds., Birkhäuser Verlag, Basel, 2010, pp. 153-179, https://doi.org/ 10.1007/978-3-0346-0152-8_9.

[Har83] A. HARTEn, High resolution schemes for hyperbolic conservation laws, J. Comput. Phys., 49 (1983), pp. 357-393.

[IR09] F. Ismail AND P.L. RoE, Affordable, entropy-consistent Euler flux functions II: Entropy production at shocks, J. Comput. Phys., 228 (2009), pp. 5410-5436.

[KL89] H.-O. Kreiss And J. Lorenz, Initial Boundary Value Problems and the Navier-Stokes Equations, Academic Press, New York, 1989.

[PCFN16] M. Parsani, M.H. Carpenter, T.C. Fisher, and E.J. Nielsen, Entropy stable staggered grid discontinuous spectral collocation methods of any order for the compressible Navier-Stokes equations, SIAM J. Sci. Comput., 38 (2016), pp. 3129-3162, https://doi.org/10.1137/15M1043510.

[PCN15] M. PARsani, M.H. CARPEnter, ANd E.J. NiElsen, Entropy stable wall boundary conditions for the three-dimensional compressible Navier-Stokes equations, J. Comput. Phys., 292 (2015), pp. 88-113.

[SM12] M. SvÄRD AND S. MishrA, Entropy stable schemes for initial-boundary-value conservation laws, Z. Angew. Math. Phys., 63 (2012), pp. 985-1003.

[SN08] M. SVÄRD AND J. NORDSTRÖM, A stable high-order finite difference scheme for the compressible Navier-Stokes equations, no-slip wall boundary conditions, J. Comput. Phys., 227 (2008), pp. 4805-4824.

[SO14] M. SvÄRD AND H. ÖZCAN, Entropy stable schemes for the Euler equations with far-field and wall boundary conditions, J. Sci. Comput., 58 (2014), pp. 61-89.

[Svä12] M. SvÄRD, Third-order accurate entropy-stable scheme for initial-boundary-value conservation laws, Z. Angew. Math. Phys., 63 (2012), pp. 599-623.

[Svä15] M. SvÄRD, Weak solutions and convergent numerical schemes of modified compressible Navier-Stokes equations, J. Comput. Phys., 288 (2015), pp. 19-51.

[Svä16] M. Svärd, A convergent numerical scheme for the compressible Navier-Stokes equations, SIAM J. Numer. Anal., 54 (2016), pp. 1484-1506, https://doi.org/10.1137/ 15M1010361.

[Tad03] E. TADMOR, Entropy stability theory for difference approximations of nonlinear conservation laws and related time-dependent problems, Acta Numer., 12 (2003), pp. 451-512.

[Tre13] W.F. Trench, Introduction to Real Analysis, Open Textbook Initiative, American Institute of Mathematics, 2013, https://aimath.org/textbooks/approved-textbooks/ trench/.

Copyright (c) by SIAM. Unauthorized reproduction of this article is prohibited. 